\title{
Effects of Physical Aging on Polarization-Induced Photodimerization in the Glassy Region of Poly(methyl methacrylate)
}

\author{
Youichi Yamaguchi, Takahiro OKamoto, Osamu Urakawa, \\ and Qui TRAN-ConG ${ }^{\dagger}$ \\ Department of Polymer Science and Engineering, \\ Kyoto Institute of Technology, \\ Matsugasaki, Kyoto 606-8585, Japan
}

(Received October 8, 1997)

\begin{abstract}
Intramolecular photodimerization of the photochromic molecule 9-hydroxymethyl-10-[(1-naphthyl methoxy)methyl]anthracene (HNMA) was investigated by ultraviolet-visible (UV-VIS) spectroscopy in the glassy region of poly(methyl methacrylate) (PMMA) under different aging conditions. The PMMA matrix was annealed for different time intervals in the glassy region after quenching from $140^{\circ} \mathrm{C}\left(37.8^{\circ} \mathrm{C}\right.$ above the glass transition temperature $\left.\left(T_{\mathrm{g}}\right)\right)$. By following the photodimerization upon irradiation with linearly polarized light, it was found that the reaction kinetics of HNMA reflects the rearrangements of local free-volumes of the matrix. The maximum efficiency $\left(\eta_{\max }\right)$ of the reaction induced by linearly polarized light decreases with increasing aging time $\left(t_{\mathrm{a}}\right)$. Under aging above $60^{\circ} \mathrm{C}, \eta_{\max }$ decreased monotonously with aging time, whereas it did not change systematically for a PMMA aged at $40^{\circ} \mathrm{C}$. The secondary relaxation of the PMMA matrix may thus play a key role in the aging process at low temperature. On the other hand, the reaction kinetics of HNMA can be well expressed by the sum of two exponential functions of irradiation time and a baseline. The two fractions of these decay processes are significantly affected by aging time whereas the influence on the two reaction rates is less remarkable. The baseline increases with increasing aging time, reflecting shrinkage of samples under aging. These experimental results are discussed in relation to the aging data obtained by other techniques such as dielectric relaxation and dynamic mechanical measurements. KEY WORDS Intramolecular Photodimerization / Poly(methyl methacrylate) / Linearly Polarized Light / Glass Transition / Secondary Relaxation / Physical Aging / Free-Volume Distribution /
\end{abstract}

Polymer solids often fall into thermodynamic nonequilibrium when cooled into the glassy state through glass transition temperatures $\left(T_{\mathrm{g}}\right)$. This situation arises from the fact that the system apparently vitrifies before reaching the "true" $T_{\mathrm{g}}$ due to extremely slow diffusion of polymer chains upon cooling. As a consequence, the glassy polymers undergo extremely slow relaxation below $T_{\mathrm{g}}$ to return to equilibrium. In practice, these relaxation processes, known as physical aging, ${ }^{1}$ play an important role in the control of polymer physical properties.

Dynamic mechanical measurements, ${ }^{2}$ dielectric relaxation, ${ }^{3}$ differential scanning calorimetry (DSC) ${ }^{4}$ are used to investigate physical aging of polymers. More recently, molecular probes have been used to monitor changes in the local environment of polymer solids undergoing physical aging. These techniques include reaction kinetics of photoisomerization of azobenzene,,$^{5-7}$ decay of second harmonic generation (SHG) signals ${ }^{8}$ and fluorescent probes with intramolecular rotations. ${ }^{9-10}$ The data show that the free-volume distribution of glassy polymers is modified during aging. Practically, the variation in the local environment of polymer solids used for photonics or for optical recording purposes may seriously affect the long-term durability of materials.

In the past few years, we have used polarizationinduced photochemical reactions for investigating extremely slow reorientational relaxation of smallmolecule dopants in the glassy state of poly(methyl methacrylate) ${ }^{11}$ where the spatial distribution of photochemical reactions in glassy polymer matrices can be

\footnotetext{
† To whom correspondence should be addressed.
}

generated and controlled by irradiation with linearly polarized light. ${ }^{12}$ According to recent theoretical and experimental results, the growth of the optical anisotropy generated by linearly polarized light is determined by the ratio of the reorientational relaxation time of dopants and reaction rate constant. ${ }^{13}$ It is also found that the experimental results are strongly affected by thermal history of the samples during preparation. Therefore, it is necessary to systematically investigate the correlations between physical aging of polymer matrix and photochemical reactions of molecular dopants under a variety of conditions.

In this paper, the effects of aging temperatures as well as of aging time on the behavior of these photoselective reactions are examined by following the kinetics and the induction efficiency of the reactions upon irradiation with linearly polarized light. Subsequently, these experimental results are compared with data obtained previously by complimentary experimental techniques such as dynamic mechanical measurements and dielectric relaxation. Finally, the contribution of the secondary $(\beta)$ relaxation process of the polymer matrix to the aging process is discussed.

\section{EXPERIMENTAL}

\section{Samples}

The polymer matrix used in this work was poly(methyl methacrylate) (PMMA, $M_{w}=1.78 \times 10^{5}$, Wako Chemicals Inc.) purified by precipitating twice using toluene/ methanol mixtures. The glass transition temperature $\left(T_{\mathrm{g}}\right)$ of this PMMA is $102.2^{\circ} \mathrm{C}$ as measured by differential scanning calorimetry (DSC, Mac Science 3100) with a heating rate of $5^{\circ} \mathrm{Cmin}^{-1}$. The photochromic molecule 


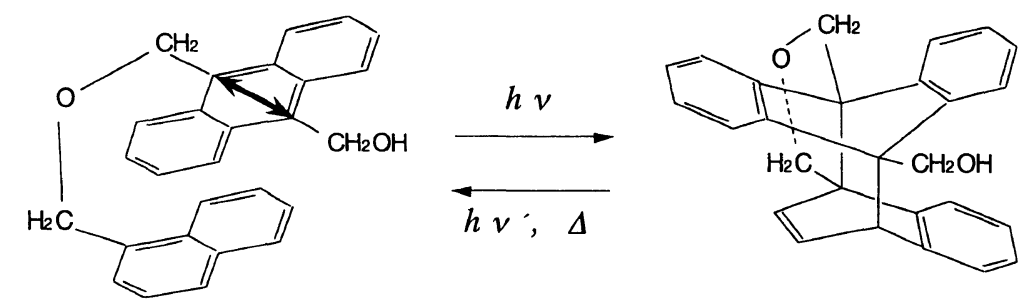

Figure 1. Chemical structures and intramolecular photodimerization of 9-hydroxymethyl-10-[(1-naphthyl methoxy) methyl]anthracene (HNMA).

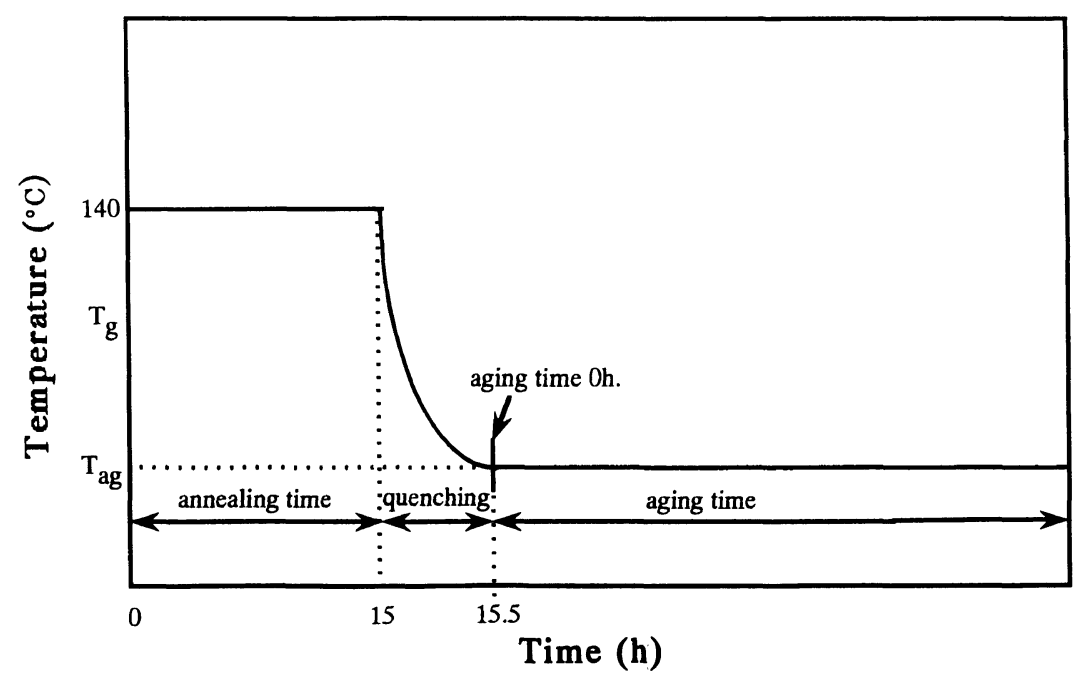

Figure 2. Temperature program for physical aging.

9-hydroxymethyl-10-[(1-naphthyl methoxy) methyl] anthracene (HNMA) was prepared and purified as reported previously. ${ }^{14}$ The chemical structure of HNMA and its intramolecular photodimerization are shown in Figure 1. The concentration of HNMA in PMMA was adjusted to $2.5-3.0 \times 10^{-3} \mathrm{M}$ by solvent casting. The sizes of the PMMA film doped with HNMA used for the irradiation experiments was $(13 \mathrm{~mm} \times 9 \mathrm{~mm} \times 0.2$ $\mathrm{mm})$. The samples were dried under vacuum at $170^{\circ} \mathrm{C}$ over 2 days to remove solvent prior to the experiments. In order to set the initial conditions, all samples were kept at $140^{\circ} \mathrm{C}$, i.e., $37.8^{\circ} \mathrm{C}$ above $T_{\mathrm{g}}$, over $15 \mathrm{~h}$ and then quenched by quickly transferring the samples from $140^{\circ} \mathrm{C}$ to a heating block preset at a given temperature. 80,60 , and $40^{\circ} \mathrm{C}$ were chosen as the aging temperatures $\left(T_{\mathrm{ag}}\right)$. The temperature diagram for the aging process at an arbitrary temperature $T_{\mathrm{ag}}$ is shown in Figure 2.

\section{Instruments}

The light source used in this work was a mercury lamp (250W, Ushio Electrics, Japan) with intensity adjustable up to $3.0 \mathrm{~mW} \mathrm{~cm}^{-2}$. UV light with wavelengths mainly at $365 \mathrm{~nm}$ was converted to linearly polarized light using a polarizer (Polaroid, $\mathrm{HNP}^{\prime} \mathrm{B}$ ) after having passed through a convex lens for focussing the beam at the sample. Changes in the absorbance of the anthracene moieties with irradiation time were monitored at 394.5 nm using a UV-VIS spectrometer (UV-1600, Shimadzu, Japan) connected to a Macintosh computer for data access. Sample temperature was controlled within a precision of $\pm 0.5^{\circ} \mathrm{C}$ by a temperature controller (Okura Electrics Inc., Model EC-5500S). Data analysis was performed by the nonlinear least-square method using

Polym. J., Vol. 30, No. 5, 1998 the software Igor on a Macintosh computer.

\section{Data Analysis}

From two polarized components of absorbance, the induction efficiency $\eta(t)$ and the total absorbance $O D(t)$ of HNMA were calculated and analyzed. The former defined below, expresses the growth of optical anisotropy in the sample under irradiation with linearly polarized light.

$$
\eta(t)=\frac{\left[O D_{\perp}(t)-O D_{\|}(t)\right]}{O D_{0}}
$$

where $O D_{\perp}(t)$ and $O D_{\|}(t)$ are absorbance measured in directions perpendicular and parallel to the exciting polarization $(\boldsymbol{E})$, respectively. $O D_{0}$ is the initial absorbance of HNMA obtained before irradiation.

The total absorbance $O D(t)$ defined by eq 2 corresponds to the absorption of the sample obtained under irradiation with unpolarized light ${ }^{15}$ :

$$
O D(t)=\left[O D_{\|}(t)+2 O D_{\perp}(t)\right]
$$

The reaction rates of HNMA in PMMA can be obtained by fitting these decay curves to appropriate functions discussed later.

\section{RESULTS}

Influence of Thermal History of PMMA on PolarizationSelective Photodimerization of HNMA

The photodimerization kinetics and the induction efficiency $\eta(t)$ of HNMA incorporated in PMMA are shown as an example in Figure 3 for two different ag- 

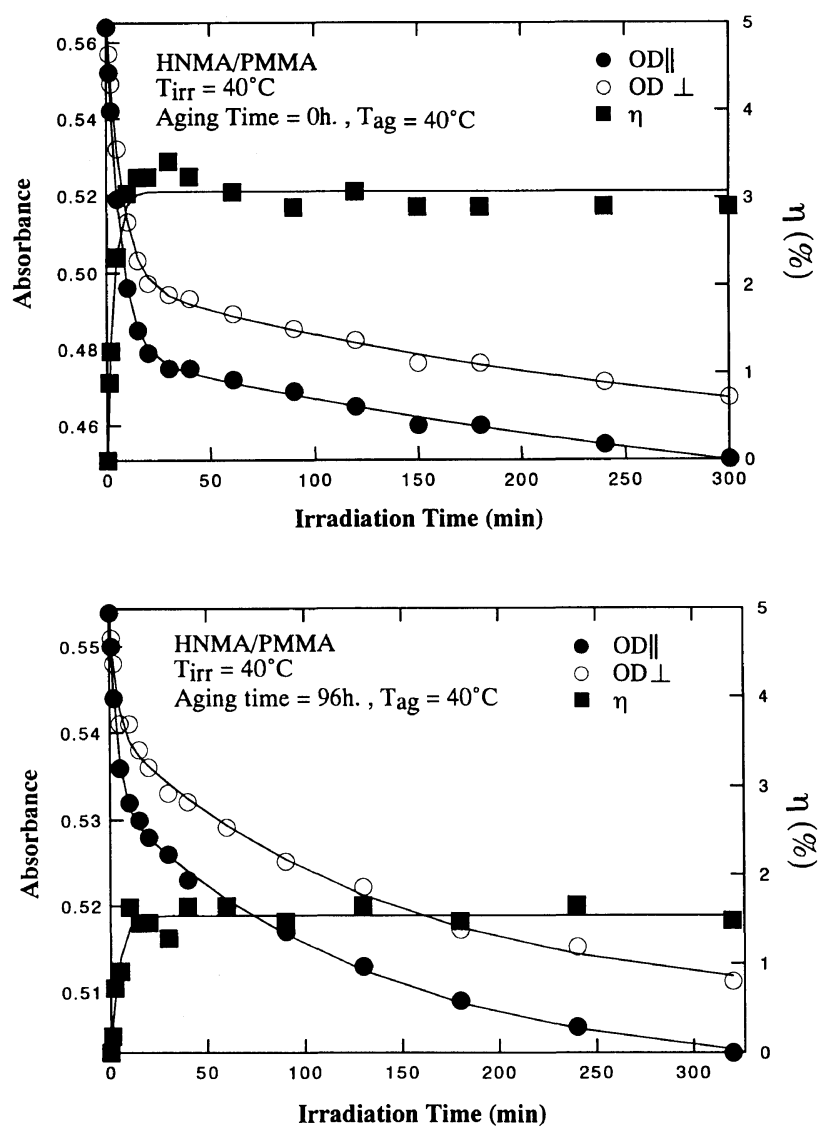

Figure 3. Polarized absorbance and induction efficiency $\eta(t)$ of HNMA in PMMA irradiated at $40^{\circ} \mathrm{C}$ after aging at $40^{\circ} \mathrm{C}$. Upper, aging time $0 \mathrm{~h}$; lower, aging time $96 \mathrm{~h}$.

ing conditions under irradiation at $40^{\circ} \mathrm{C}$. These two samples were quenched from 140 to $40^{\circ} \mathrm{C}$ after annealing at $140^{\circ} \mathrm{C}$ for $15 \mathrm{~h}$ according to the diagram shown in Figure 2. As seen from the decay curves of absorbance illustrated in Figure 3, the conversion of intramolecular photodimerization obtained in the case of quenching without aging was higher than that of the sample aged over $96 \mathrm{~h}$ at the same temperature. Furthermore, the induction efficiency $\eta(t)$ was much larger than the aged PMMA. As reported previously, the conversion of the photodimerization of HNMA is controlled by intramolecular conformational transition, a rotational diffusion-controlled process. ${ }^{16}$ It is also known from recent theoretical studies that the induction efficiency $\eta(t)$ is determined by the ratio of the reaction rate to the reorientational relaxation time. ${ }^{13}$ Therefore, the experimental results shown in Figure 3 strongly indicate that changes in the local structures of PMMA associated with the aging process play important roles in intramolecular photodimerization as well as reorientational relaxation of HNMA. In order to elucidate the contribution of these physical aging processes, PMMA containing HNMA was aged at three temperatures 80,60 , and $40^{\circ} \mathrm{C}$ below its $T_{\mathrm{g}}$. The aging time is varied between 0 to $96 \mathrm{~h}$ depending upon the aging temperature. Figure 4 shows the change in the total absorbance $O D(t)$ defined in eq 2 with irradiation time at different aging times $\left(t_{\mathrm{a}}\right)$ for PMMA aged at $60^{\circ} \mathrm{C}$. As irradiation time increases, the total absorbance $O D(t)$ decreases non-exponentially, indicating that photodimerization of HNMA takes

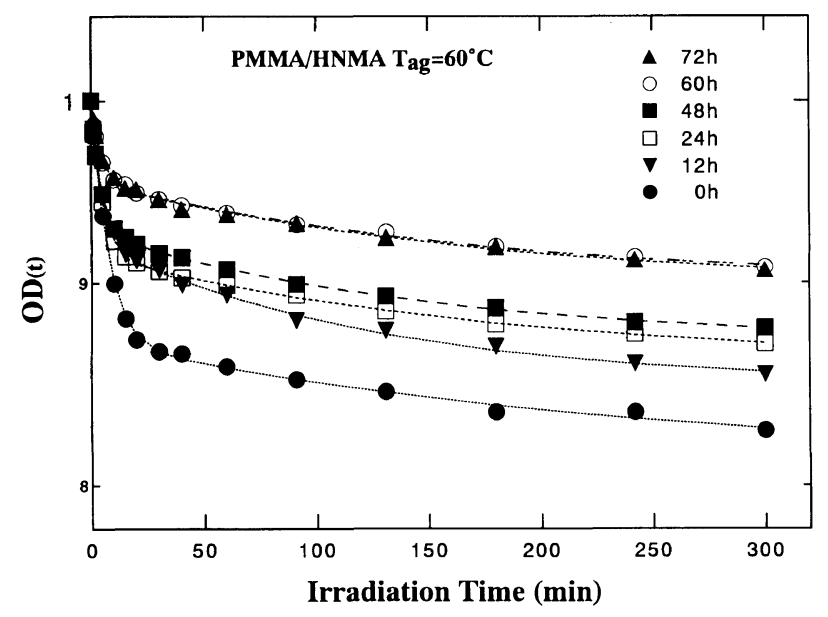

Figure 4. Effects of aging time on the total absorbance $O D(t)$ of HNMA in a PMMA matrix aged at $60^{\circ} \mathrm{C}$. Numbers in the figure indicate aging times.

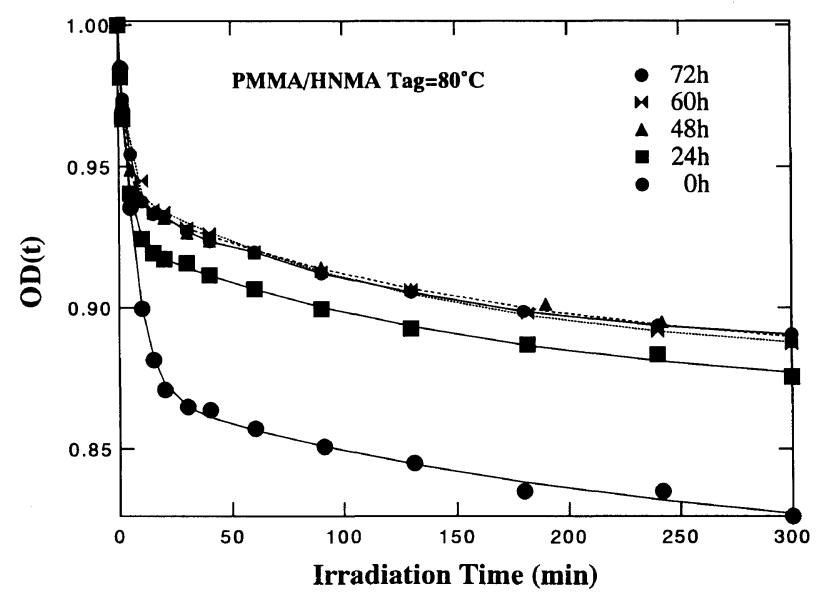

Figure 5. Effects of aging time on the total absorbance $O D(t)$ of HNMA in a PMMA matrix aged at $80^{\circ} \mathrm{C}$. Numbers in the figure indicate aging times.

place inhomogeneously. In the initial stage, the reaction quickly takes place and proceeds very slowly later. Eventually, $O D(t)$ tends to approach a limiting value, $O D_{\infty}$, indicating that HNMA does not react completely in the aged sample. Similar results were observed with PMMA aged at $80^{\circ} \mathrm{C}$ as illustrated in Figure 5 where changes in the total absorbance $O D(t)$ with irradiation time seem to reach equilibrium with shorter aging time. The kinetics shown in Figures 4 and 5 reveal the existence of a free-volumes distribution in the PMMA samples because the intramolecular photo-dimerization of diphant molecules is sensitive to the local environment in bulk polymer matrix. ${ }^{16}$ Taking into account a free-volume distribution around HNMA in the PMMA matrix, the decay of the total absorbance $O D(t)$ was fitted to the following model equation:

$$
O D(t)=F_{\mathrm{f}} \exp \left(-k_{\mathrm{f}} t\right)+F_{\mathrm{s}} \exp \left(-k_{\mathrm{s}} t\right)+O D_{\infty}
$$

where $k_{\mathrm{f}}$ and $k_{\mathrm{s}}$ are respectively the rates of the fast and slow reactions. $E_{\mathrm{f}}$ and $F_{\mathrm{s}}$ are the corresponding fractions. Here $\mathrm{f}$ and $\mathrm{s}$ stand for fast and slow. As described above, the limiting absorbance $O D_{\infty}$ is introduced into eq 3 to express the fact that all the HNMA molecules dispersed in PMMA cannot completely react 


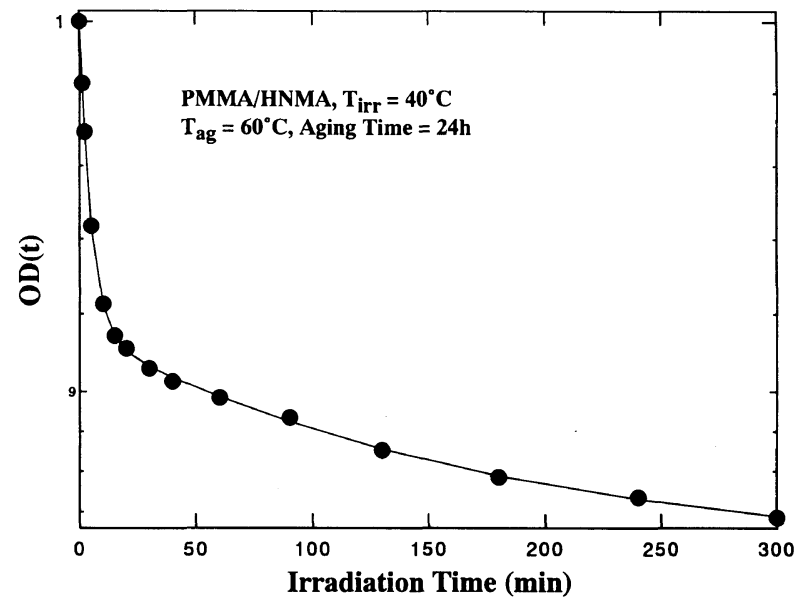

Figure 6. Total absorbance $O D(t)$ of HNMA in a PMMA aged at $60^{\circ} \mathrm{C}$ over $24 \mathrm{~h}$. The experimental temperature is $40^{\circ} \mathrm{C}$. The fitting parameters are $F_{\mathrm{s}}=5.64 \times 10^{-2} ; \quad F_{\mathrm{f}}=8.41 \times 10^{-2} ; \quad k_{\mathrm{s}}=5.74 \times 10^{-3}$ $\min ^{-1} ; k_{\mathrm{f}}=0.21 \mathrm{~min}^{-1}$.

upon irradiation over long time. An example for the curve fitting using eq 3 is shown in Figure 6 for the total absorbance $O D(t)$ of HNMA monitored at $394.5 \mathrm{~nm}$ in a PMMA aged at $60^{\circ} \mathrm{C}$ over $24 \mathrm{~h}$. Because the reaction of HNMA is sensitive to free-volume of the local environment, the fast and slow components of $O D(t)$ reflect the reactions of HNMA in the regions characterized by $v_{\mathrm{f}}>v_{\text {HNMA }}$ and $v_{\mathrm{f}} \leq v_{\text {HNMA }}$. Here, $v_{\mathrm{f}}$ is the free-volume of PMMA segments and $v_{\text {HNMA }}$ is the minimal volume required for the photodimerization of HNMA. Physically, $F_{\mathrm{f}}$ and $k_{\mathrm{f}}$ can be interpreted as the fraction and the reaction rate in the region with the free volumes much larger than $v_{\text {HNMA }}$, whereas $F_{\mathrm{s}}$ and $k_{\mathrm{s}}$ are those of HNMA in the regions with free volumes smaller or comparable to $v_{\mathrm{HNMA}}$. It is worth noting that because the Kohlrausch-Williams-Watts (KWW) kinetics, ${ }^{17}$ i.e., the stretched exponential function, does not provide a satisfactory fit for these data, we have to use the multi-exponential function approach based on the free-volume concept explained above. The validity of the fitting using the particular model function given in eq 3 is probably due to the fixed size of the molecular probe HNMA which serves as a "ruler" for measuring smaller and larger free-volumes compared to the effective volume $v_{\mathrm{HNMA}}$ in the PMMA matrix. In other word, HNMA reflects discretely the continuous distribution of free-volumes of PMMA. The presence of the limiting total absorbance $O D_{\infty}$ suggests that there exists in the aged sample a certain fraction of HNMA trapped in the regions with free volumes much smaller than the threshold $v_{\mathrm{f}}^{*}=v_{\text {HNMA }}$ required for the intramolecular photodimerization. The non-exponential decay of $O D(t)$ observed in Figures 4 and 5 reflects the rearrangements of the free-volume distribution of PMMA with aging. This conclusion is supported by the results in Figure 7 where the limiting total absorbance $O D_{\infty}$ is plotted against aging time for three PMMA samples annealed at different temperatures. For the sample aged at 60 and $80^{\circ} \mathrm{C}, O D_{\infty}$ gradually increases and tends to reach equilibrium after $c a .60 \mathrm{~min}$ of aging. Namely, $O D(t)$ of the PMMA samples aged at temperatures closer to the glass transition temperature $\left(T_{\mathrm{g}}\right)$ reach the stationary state with shorter irradiation time. At 80 and $60^{\circ} \mathrm{C}$, the

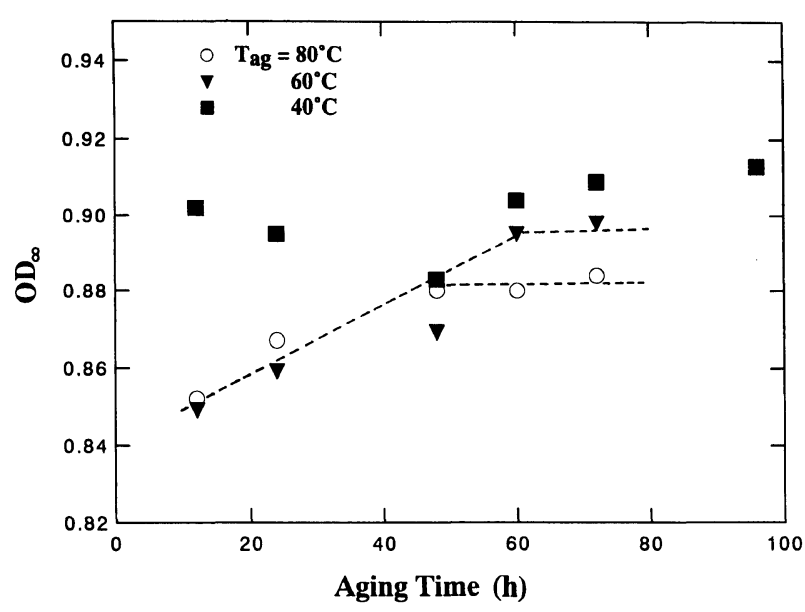

Figure 7. Dependence of the limiting total absorbance $O D_{\infty}$ on the aging time at different aging temperatures.

two fractions $F_{\mathrm{f}}$ and $F_{\mathrm{s}}$ decrease with increasing aging time, suggesting that the intramolecular photodimerization of HNMA is affected by shrinkage associated with aging. On the other hand, $k_{\mathrm{f}}$ does not significantly change with aging time whereas $k_{\mathrm{s}}$ which characterizes the slow reaction process, slightly decreases with increasing aging time and is somewhat sensitive to aging temperatures. As an example for the aging time dependence of these kinetic parameters, the data obtained for a PMMA sample aged at $60^{\circ} \mathrm{C}$ over different time intervals are shown in Figures 8 and 9. Because the time required for monitoring the decay of absorbance of HNMA is $c a$. $5 \mathrm{~h}$, the data obtained with the shortest aging time $(12 \mathrm{~h})$ for a PMMA at the highest aging temperature $\left(80^{\circ} \mathrm{C}\right)$ may be affected by the aging process ongoing during measurement. The kinetics obtained at higher temperatures are thus not shown here. It has been suggested that the optimal time required to accomplish the measurements may be $1 / 10$ of the aging time. ${ }^{1}$ From these kinetic data, it can be concluded that the distribution of free-volume in PMMA becomes narrow with aging as observed through the intramolecular photodimerization kinetics of HNMA. This is consistent with the results obtained by other molecular probes. ${ }^{6,7,10}$ On the other hand, the kinetics obtained with PMMA aged at $40^{\circ} \mathrm{C}$ does not show systematic variation with aging time. As an example, the variation of the total absorbance $O D(t)$ with aging time at this temperature is shown in Figure 10 where the consumption of HNMA under irradiation with linearly polarized light does not vary systematically with aging time. Similar behavior was already seen in Figure 7 for the limiting total absorbance $O D_{\infty}$ and also for the limiting induction efficiency $\eta_{\max }$ as described below. McKenna and Kovacs reported that the torque-relaxation data obtained for PMMA aging at low temperatures $\left(40\right.$ and $60^{\circ} \mathrm{C}$ ) cannot be superposed for any combinations of vertical and horizontal shifts with respect to the aging time axis. ${ }^{18}$ On the other hand, relaxation data obtained for the same PMMA aged at temperatures close to $T_{\mathrm{g}}\left(80^{\circ} \mathrm{C}\right)$ can be superimposed to generate a master curve by horizontal shifts along the axis of aging time as originally proposed by Struick for a large number of glassy polymers. ${ }^{1}$ Similar failure of the "time-temperature superposition" concept in 

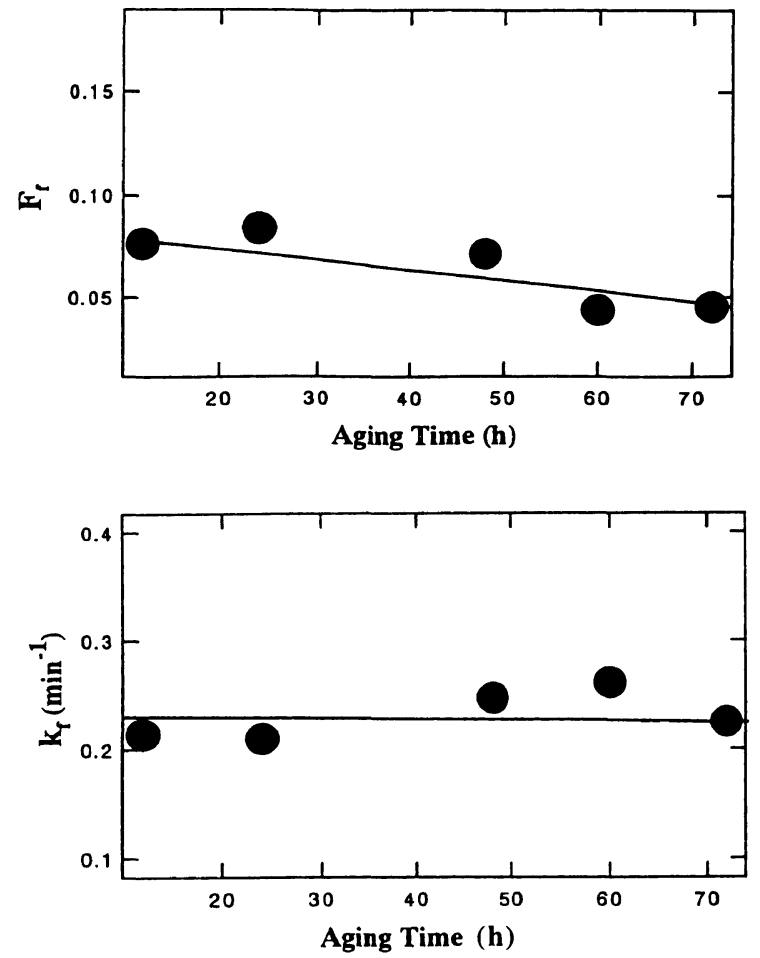

Figure 8. Aging-time dependence of $F_{\mathrm{f}}$ and $k_{\mathrm{f}}$ for the fast component of the total absorbance $O D(t)$ monitored at $60^{\circ} \mathrm{C}$.
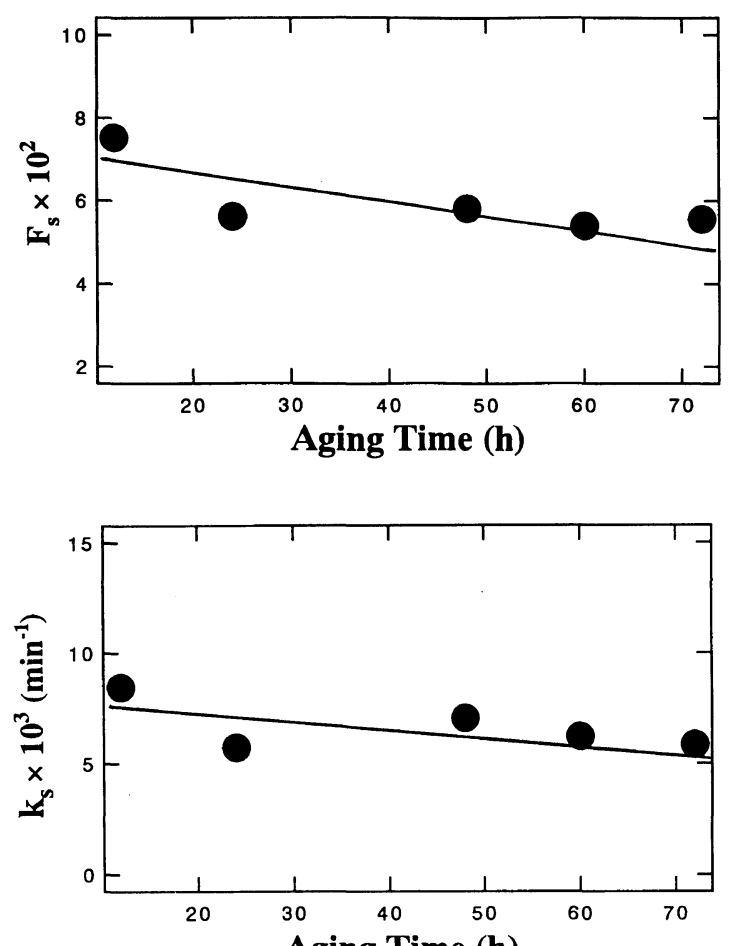

Aging Time (h)

Figure 9. Aging-time dependence of $F_{\mathrm{s}}$ and $k_{\mathrm{s}}$ for the slow component of the total absorbance $O D(t)$ monitored at $60^{\circ} \mathrm{C}$.

glassy PMMA under aging at ambient temperatures has been also reported by dielectric relaxation ${ }^{3}$ and dynamic mechanical measurements. ${ }^{2}$ This failure in the contruction of "universal" curves by either horizontal or vertical shifts has been attributed by these authors to the contribution of the secondary $(\beta)$ relaxation of PMMA. In previous studies on intramolecular photodimerization

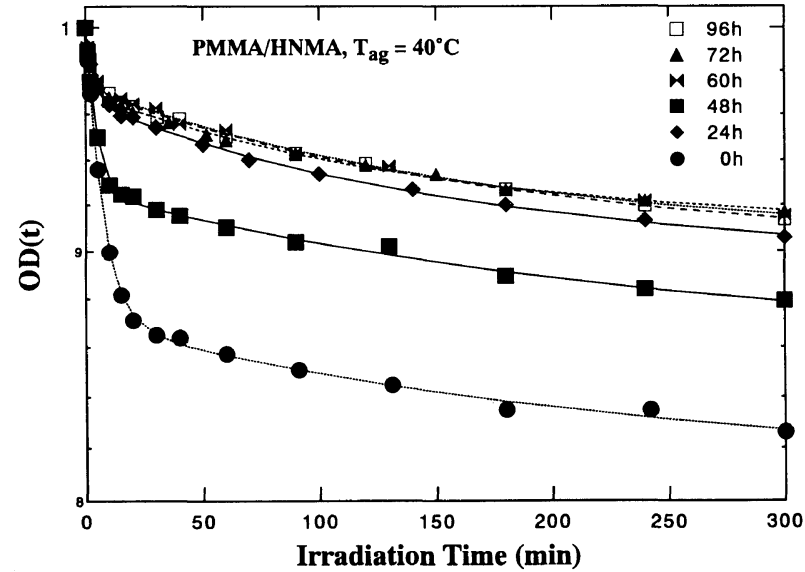

Figure 10. Effects of aging time on the total absorbance $O D(t)$ of HNMA observed for a PMMA matrix physically aged at $40^{\circ} \mathrm{C}$. Numbers in the figure are aging times.

of the so-called diphant molecules, it was found that the reaction kinetics of HNMA and its analog bis(9-anthryl methyl) ether (BAME) were significantly affected by the $\beta$-relaxation of PMMA ${ }^{19}$ and polystyrene. ${ }^{16}$ Furthermore, the induction efficiency of HNMA in PMMA exhibits a maximum in the vicinity of the transition temperature for the $\beta$ process $\left(\mathrm{ca} .40-50^{\circ} \mathrm{C}\right) .{ }^{14}$ From these experimental results, we conclude that the nonsystematic variation of the kinetics data with aging time observed at $40^{\circ} \mathrm{C}$ is likely due to the $\beta$-relaxation.

\section{Effects of Aging Temperatures on the Induction Efficiency of HNMA in PMMA}

From the two polarized components of absorbance measured in directions parallel and perpendicular to the exciting polarization $(\boldsymbol{E})$, the induction efficiency defined in eq 1 was obtained for HNMA in PMMA aged at 80 , 60 , and $40^{\circ} \mathrm{C} . \eta(t)$ obtained for a PMMA quenched from 140 to $40^{\circ} \mathrm{C}$ with and without aging is shown as an example in Figure 3. The irradiation time dependence of the induction efficiency $\eta(t)$ was fit to the following equation ${ }^{13}$ :

$$
\eta(t)=\eta_{\max }\left(1-\mathrm{e}^{-K t}\right)
$$

where $\eta_{\max }$ and $K$ are respectively the limiting induction efficiency and the growth rate of the optical anisotropy generated by irradiation with linearly polarized light.

Figure 11 shows the limiting induction efficiency $\eta_{\max }$ obtained for PMMA aged in different time intervals at 80,60 , and $40^{\circ} \mathrm{C}$. For 60 and $80^{\circ} \mathrm{C}, \eta_{\max }$ decreases monotonously with aging time. Though the magnitudes of these $\eta_{\max } \mathrm{s}$ are not large, the behavior on aging at these two temperatures is consistent with the total absorbance $O D(t)$ obtained at the same temperatures in Figures 4 and 5. For the sample aged at $60^{\circ} \mathrm{C}, \eta_{\max }$ gradually decreases with increasing aging time and eventually approaches equilibrium after $60 \mathrm{~h}$. This is consistent with the behavior of $O D(t)$ in Figure 4 where the limiting total absorbance becomes insensitive to the aging time after $60 \mathrm{~h}$. On the other hand, $\eta_{\max }$ does not monotonously decrease with aging time for PMMA aged at $40^{\circ} \mathrm{C}$, suggesting the contribution of the $\beta$ relaxation of the PMMA matrix. Recently, general solutions for the polarized-induced photochemical reac- 

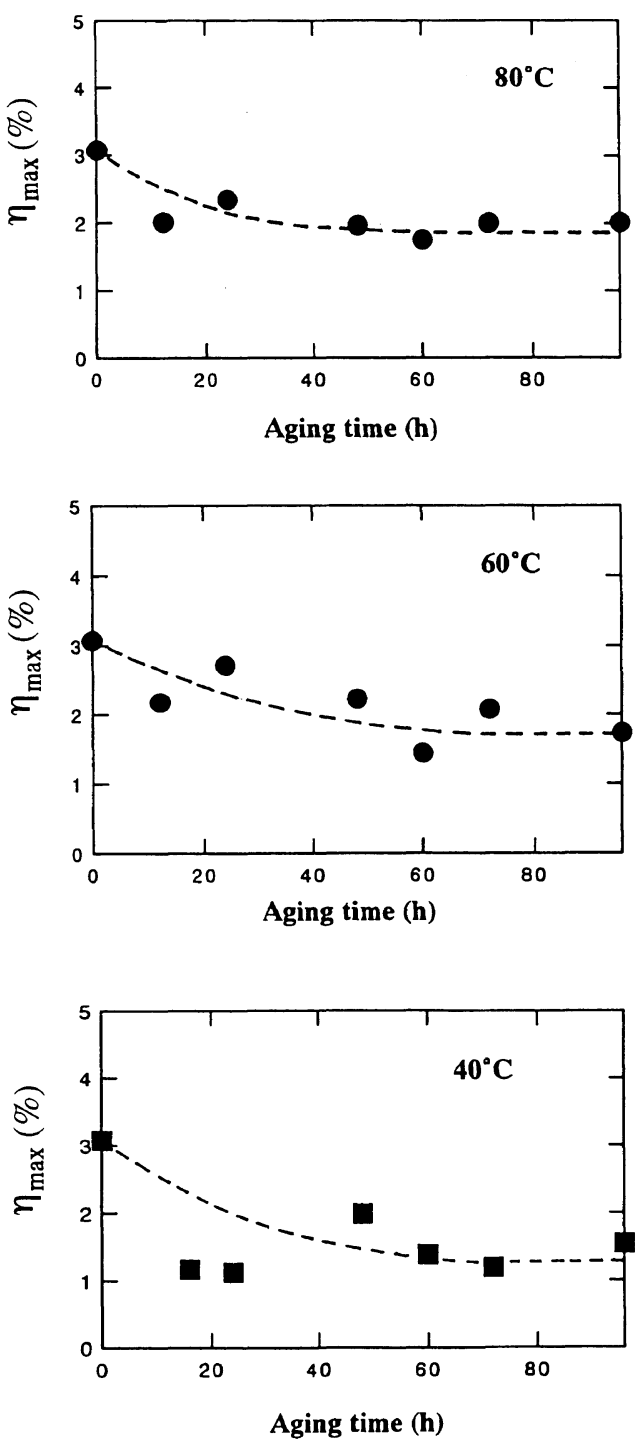

Figure 11. Aging-time dependence of the maximum induction efficiency $\eta_{\max }$ obtained at different aging temperatures. Upper, $80^{\circ} \mathrm{C}$; middle, $60^{\circ} \mathrm{C}$; lower, $40^{\circ} \mathrm{C}$.

tions have been obtained by analytically solving a rotational diffusion equation containing a chemical reaction term. ${ }^{13}$ According to these results, the equilibrium induction efficiency $\eta_{\max }$ is determined by the ratio of reaction rate to reorientational relaxation time of the dopant molecule. From the aging time dependence of the total absorbance $O D(t)$ in Figures 4 and 5 , it can be concluded that the decrease in the reaction conversion as well as the reaction rate $k_{\mathrm{s}}$ with aging time of HNMA is partially responsible for the decay of $\eta_{\max }$ in Figure 11. We have attempted to directly measure the reorientational relaxation of HNMA in PMMA under aging at different temperatures using the annealing-after-irradiation method. ${ }^{11,19}$ However, the induction efficiency $\eta$ measured after aging was not large enough (ca. 3\%) for obtaining accurate kinetic data from the annealing-after-irradiation experiments. To obtain more reliable data for the reorientational relaxation of HNMA under a given experimental condition, experiments using various aging conditions are in progress.

\section{SUMMARY}

Intramolecular photodimerization of a diphant-type molecule, HNMA in a PMMA matrix was investigated under different aging conditions using polarizationinduced reaction kinetics. By changing aging time and aging temperature, the following were found:

1) The reaction kinetics and the optical anisotropy generated by irradiation with linearly polarized light are strongly affected by aging temperature and aging time.

2) The reaction kinetics can be well expressed by the sum of two exponential functions of aging time and a baseline. The latter increases and tends to approach equilibrium at long aging time. The fractions $F_{\mathrm{f}}$ and $F_{\mathrm{s}}$ of the fast and slow components of the reactions decrease with increasing aging time, indicating intramolecular photodimerization of HNMA to be sensitive to shinkage of PMMA samples upon aging.

3) Changes in the two rate constants $k_{\mathrm{f}}$ and $k_{\mathrm{s}}$ with aging time are not so significant though the slow reaction rate $k_{\mathrm{s}}$ decreases with aging time and is slightly sensitive to temperature. The distribution of the free volumes located around HNMA in PMMA may thus be narrower with aging.

4) The reaction kinetics as well as the induction efficiency obtained in the aging experiments at low temperatures are in agreement with dielectric and dynamic mechanical relaxation data, revealing the remarkable roles of the secondary $(\beta)$ relaxation of PMMA under aging.

Experiments using polymer matrices with the secondary relaxation temperatures lower than that of PMMA are in progress to evaluate the affects of the $(\beta)$ relaxation on the aging process of glassy polymers.

Acknowledgments. Financial support from the Ministry of Education, Culture, and Science of Japan via Grants-in-Aid No. 08218234 for the Priority-AreaResearch "Photoreaction Dynamics" is greatly appreciated. We thank Dr. Gregory B. McKenna (Polymers Division, National Institute of Standards and Technology, Maryland, U.S.A.) for simulating discussions and for bringing ref 18 to our attention.

\section{REFERENCES}

1. L. C. E. Struik, "Physical Aging in Amorphous Polymers and Other Materials," Elsevier, Amsterdam, 1978.

2. L. Guerdoux, R. A. Duckett, and D. Froelich, Polymer, 25, 1392 (1984).

3. L. Guerdoux and E. Marchal, Polymer, 22, 1199 (1981).

4. S. R. Jong, J. S. Lee, and T.-L. Yu, Macromol. Chem. Phys., 198, 2373 (1997).

5. L. Lamarre and C. S. P Sung, Macromolecules, 16, 1729 (1983); C. S. P. Sung, L. Lamarre, and K. H. Chung, Macromolecules, 14, 1839 (1981).

6. J. G. Victor and J. M. Torkelson, Macromolecules, 20, 2241 (1987).

7. I. Mita, K. Horie, and K. Hirao, Macromolecules, 22, 558 (1989).

8. C. Y. Stacey Fu, H. S. Lackritz, D. B. Priddy, Jr., and J. E. McGrath, Macromolecules, 29, 3470 (1996).

9. E. F. Meyer, A. M. Jamieson, and R. Simha, Polymer, 31, 243 (1990).

10. J. S. Royal and J. M. Torkelson, Macromolecules, 26, 5331 (1993). 
Y. YAMAGUCHI et al.

11. H. Kanato, D. H. Hua, and Q. Tran-Cong, Macromolecules, 27, 7907 (1994).

12. Q. Tran-Cong, "Polarization-Selective Photochromic Reactions in Polymer Solids" in "Polymeric Materials Encyclopedia," Vol. 7, CRC Press, Boca Raton, FL, 1966, p 5928.

13. A. Morita and Q. Tran-Cong, Physica A, 242, 377 (1997).

14. Q. Tran-Cong, T. Kumazawa, O. Yano, and T. Soen, Macromolecules, 23, 3002 (1990)
15. R. J. Cherry, Biochim. Biophys. Acta, 559, 289 (1979).

16. H. Yoshizawa, K. Ashikaga, M. Yamamoto, and Q. Tran-Cong, Polymer, 30, 534 (1989).

17. G. Williams and D. C. Watts, Trans. Faraday Soc., 66, 80 (1971).

18. G. B. McKenna and A. J. Kovacs, Polym. Eng. Sci., 24, 1138 (1984).

19. Q. Tran-Cong, S. Chikaki, and H. Kanato, Polym. Commun., 35, 4465 (1994). 